\title{
Reduced Complexity Equalization Schemes for Zero Padded OFDM Systems
}

\author{
M. R. Bhavani Shankar and K. V. S. Hari, Senior Member, IEEE
}

\begin{abstract}
In this work, three reduced complexity equalization schemes for Zero-padded OFDM systems are described. These schemes guarantee Zero-Forcing (ZF) equalization irrespective of the channel nulls. Two of these schemes implement the minimum-norm $Z F$ equalizer efficiently using $Q R$ decomposition. In the third scheme, the channel zeros are grouped as being inside or outside or on the unit circle. These groups are then equalized sequentially in a manner so as to tackle excess noise amplification. The three schemes are compared for their computational complexity and Bit Error Rate (BER) performance. It is shown that the attractive scheme depends on the system specifications. The BER-Computations trade off occurring in the choice of the right algorithm is also highlighted.
\end{abstract}

Index Terms-Minimum-maximum phase, Orthogonal Frequency Division Multiplexing (OFDM), pseudo-inverse, Toeplitz matrices, zero padding.

\section{INTRODUCTION}

$\mathbf{T}$ HE use of Zero Padding (ZP) [1] in Orthogonal Frequency Division Multiplexing (OFDM) systems provides for equalizers that guarantee Zero Forcing Equalization (ZFEQN) for all nonzero channels. The minimum-norm Zero Forcing Equalizer (ZFE) given in (9) of [1] is one such equalizer. Henceforth, the minimum-norm equalizer is referred to as the Standard ZFE (SZFE). However, direct implementation of the pseudo inverse solution is computationally expensive [3]. Fast equalizers for $\mathrm{ZP}$ scheme are proposed in [2], which, unfortunately, do not guarantee ZFEQN for all channels. In this work we describe three low complexity schemes that yield ZFEQN for all nonzero channels. We begin with the use of QR Decomposition (QRD) [3] for efficient implementation of SZFE. Since SZFE solves a Least Squares (LS) problem involving a Toeplitz matrix, any of the fast algorithms in [4] can be used to implement it. Since all the algorithms in [4] have similar orders of complexity, we consider one of them-the BBH algorithm [6], [4]-for implementing SZFE. A recent work by Sinn et al. [5], uses an algorithm from [4] for obtaining an efficient $\mathrm{ZF}$ algorithm in a slightly different context. We also show that SZFE implemented using Householder's method [3] can be cheaper than the BBH algorithm under certain conditions. We then propose the Min-Max equalization, which is based on grouping the channel zeros as being inside, outside or on the

Manuscript received December 6, 2003; revised January 15, 2004. The associate editor coordinating the review of this manuscript and approving it for publication was Dr. Geert Leus.

The authors are with the Department of Electrical Communication Engineering, Indian Institute of Science, Bangalore, India (e-mail: bshankar@protocol.ece.iisc.ernet.in; hari@ece.iisc.ernet.in).

Digital Object Identifier 10.1109/LSP.2004.833491 unit circle and equalizing for these groups sequentially. These equalizers are evaluated for their computational complexity and Bit Error Rate (BER) performance. The choice of the attractive algorithm and the BER performance-complexity trade-off occurring therein are discussed.

\section{EQUALIZATION Using Zero PADDING}

We consider a system model similar to the one presented in [1]. Let $P$ be the number of sub-carriers and $H(z)$ be the baseband channel transfer function with degree $L$ and co-efficients $\left\{h_{n}\right\}_{n=0}^{L}(P>L)$. Let $N=P+L$ and $\underline{u}(i)$ be the data transmitted on $P$ sub-carriers (output of $P \times P$ IFFT) at $i$ th instant. Let $y(i)$ be the $N$-fold blocked received vector at $i$ th instant and $\bar{\eta}(i)$ be the $N \times 1$ Additive White Gaussian Noise vector at the receiver front-end. The synchronized receiver is assumed to know the channel perfectly. The transmitter normally pads $L^{\prime} \geq L$ zeros to $\underline{u}(i)$. For simplicity, we assume that $L^{\prime}=L$. Let $\tilde{\mathbf{H}}$ be the $N \times P$ Toeplitz matrix with $\left[h_{0}, 0, \ldots, 0\right]_{P \times 1}$ and $\left[h_{0}, h_{1}, \ldots, h_{L}, \ldots, 0\right]_{N \times 1}^{T}$ being its first row and column respectively [1]. The input-output relation and the SZFE [1] ${ }^{1}$ for such a zero padded OFDM system are given by (1) and (2), respectively, as

$$
\begin{aligned}
& \underline{y}(i)=\tilde{\mathbf{H}} \underline{u}(i)+\underline{\eta}(i) \\
& \underline{\hat{u}}(i)=\tilde{\mathbf{H}}^{\dagger} \underline{y}(i) .
\end{aligned}
$$

The computations required for direct implementation of $\tilde{\mathbf{H}}^{\dagger}$ is $O\left(N^{3}\right)$ [3]. Since FFT/IFFT operations are unitary, it suffices to have an estimate of $\underline{u}(i)$. Further, (1) and hence the proposed techniques are also valid for the general class of zero padded block transmission systems [1].

\section{IMPLEMENTING STANDARD ZFE USING QR DECOMPOSITION}

Let $\tilde{\mathbf{H}}=\mathbf{Q R}$ denote the QRD of $\tilde{\mathbf{H}}$ [3]. Since any nonzero $\tilde{\mathbf{H}}$ is full rank [1], the output of $\operatorname{SZFE}(\underline{\hat{u}}(i),(2))$ is obtained as the solution of $\mathbf{Q}^{*} \underline{y}(i)=\mathbf{R} \underline{\hat{u}}(i)$ [3]. In this section, we employ two known algorithms to find the QRD of $\tilde{\mathbf{H}}$ efficiently, so that $\underline{\hat{u}}(i)$ can be obtained as above. Complexities of the implementations are also evaluated. For brevity, operations are abbreviated as CA, CM, CD, RA, RM, RD, RCM, and RCD. Here, A stands for additions (and subtractions), $M$ for multiplications and $\mathrm{D}$ for divisions. $\mathrm{C}(\mathrm{R})$ denotes that both the operands are complex (real). RCx involves a real and a complex operand. Real complex operations are mentioned as they are cheaper than their complex counterparts. Further, for a vector $\underline{a}$, we implement

\footnotetext{
${ }^{1}$ Operations: ${ }^{T}$ denotes Transpose, ${ }^{*}$ Hermitian and $\dagger$ Pseudo inverse
} 
$(\underline{a} / b)$ as $\underline{a} c, c=(1 / b)$. Operations involving a zero at known positions (independent of $\left\{h_{n}\right\}$ ) are not counted.

\section{A. Bojanczyk, Brent, and de Hoog (BBH) Algorithm}

The BBH algorithm [6] can be simplified to exploit the sparseness of $\tilde{\mathbf{H}}$. A part of the simplified algorithm is described in [5]. The simplified algorithm, in its entirety, is described below for completeness. Let $\mathbf{A}(m: n, p: q)$ denote the sub-matrix formed by retaining rows $m$ through $n$ and columns $p$ through $q$ of a matrix $\mathbf{A}$. Let $a_{i, j}$ be the $(i, j)$ th element of $\mathbf{A}$ and $\underline{a}_{k}$ be the $k$ th element of vector $\underline{a}$.

Initialization: $r_{1,1}=\sqrt{\sum_{n=0}^{L}\left|h_{n}\right|^{2}}, \underline{s}=\mathbf{Q}(1: N, 1)=$ $(\tilde{\mathbf{H}}(1: N, 1)) /\left(r_{1,1}\right), f=\left(\tilde{\mathbf{H}}(1: L+1,1)^{*} \tilde{\mathbf{H}}(1: L+\right.$ $1,2: P)) /\left(r_{1,1}\right), \mathbf{R}(1,2: P)=f$. Initializations require a square-root, a RD, $(L(L+1)) /(2) \mathrm{CM},(L(L-1)) /(2) \mathrm{CA}$, $2(L+1) \mathrm{RM}, 2 L+1 \mathrm{RA}$ and $L+1 \mathrm{RCM}$.

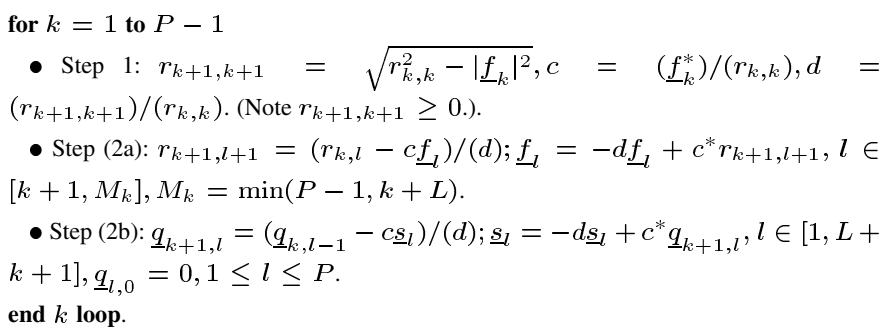

Step 3: $\underline{y}_{P \times 1}(i)=\mathrm{Q}^{*} \underline{y}(i)$. This requires $P L+(P(P+$ 1))/(2) CM and $P L+(P(P-1)) /(2) \mathrm{CA}$. The presence of only $L+k$ nonzero entries in the $k$ th column of $\mathbf{Q}$ is exploited.

Step 4: Solve $\mathbf{R} \underline{\hat{u}}(i)=\underline{y}_{P \times 1}(i)$ by Back Substitution. This step requires about $(P-L) L+(L(L-1)) /(2) \mathrm{CM},(P-$ $L) L+(L(L-1)) /(2) \mathrm{CA}$ and $P \mathrm{CD}$ when the low bandwidth $(L, L<P)$ [3] of $\mathbf{R}$ and positivity of $r_{i, i}$ are exploited.

Step $1(\forall k)$ requires $4(P-1) \mathrm{RM}, 2(P-1) \mathrm{RA}, P-1 \mathrm{RD}$, $P-1$ RCM and $P-1$ square roots. Since $r_{i, i}$ are taken to be positive, $d$ 's are real resulting in RCM. Step (2a) $(\forall k)$ requires around $(P-L-1)(2 L-1)+L(L-1) \mathrm{CM},(P-L-1)(2 L-1)+$ $L(L-1) \mathrm{RCM}, P-1 \mathrm{RD}$, and $2(P-L-1)(L-1)+L(L-1)$ CA. Further, Step $(2 \mathrm{~b})(\forall k)$ requires about $2(P-1) L+\left(P^{2}-1\right)$ CM, $2(P-1) L+\left(P^{2}-1\right)$ RCM and $2(P-1) L+(P-1)(P-2)$ CA (computing $\underline{s}$ even for $k=P-1$ ). The total number of floating point operations (flops) [3] required by BBH method is approximately $O(P L)+O\left(P^{2}\right)$.

\section{B. Householder's Method}

Apart from the fast algorithms, Householder's, Givens and other classical methods [3] can also be used to obtain QRD of $\tilde{\mathbf{H}}$. We use the Householder's method as a representative algorithm. Further, Householder's method is shown to perform QRD efficiently for some cases [3]. The Householder's algorithm for finding full rank LS solution in [3] is used here with simple modifications to account for the low bandwidth of $\tilde{\mathbf{H}}$. Hence, we do not describe the algorithm here. Further, the implementation is as explained in [3]. Obtaining the parameters of Householder's matrix $(\forall P)$ requires approximately $O(P L)$ RM, $O(P L)$ RA, $O(P)$ Square roots, $O(P)$ RCM and $O(P)$ CD. Obtaining $\mathbf{R}$ and $\mathbf{Q}^{*} y(i)$ requires about $L^{3}+(P-L-$
1) $\left(2 L^{2}-L+1\right)+(L(L+1)) /(2)+2 L P \mathrm{CM}, L^{2}+(P-L-$ 1) $\left(2 L^{2}-L+1\right)+(L(L-1)(2 L+1)) /(2)+(2 L+1) P C A$ and $(P-L) 2 L+L^{2}+(L(L-1)) /(2)+P$ RCM. RCM arise because $\beta$ [3] used is real. Finally, back substitution requires $P \mathrm{CD}$, $(P-L) L+(L(L-1)) /(2) \mathrm{CM}$ and $(P-L) L+(L(L-1)) /(2)$ CA because of the low bandwidth of $\mathbf{R}$. So this method approximately requires $O\left(P L^{2}\right)+O(P L)$ flops. The fact that this algorithm uses sparseness effectively (for some cases), better than $\mathrm{BBH}$, to reduce computations is shown in Section V.

\section{Min-MaX EQUALIZATION}

To motivate this scheme, assume (1) noise-free case, (2) channel has no zeros on the unit circle and (3) $h_{0} \neq 0$. Let $y(i)_{P}$ be the first $P$ entries of $y(i)$ and $\tilde{\mathbf{H}}_{0}$ be the leading $P \times P$ Lower Triangular Toeplitz (LTT) sub-matrix of $\tilde{\mathbf{H}}$. Then $\underline{u}(i)$ can be easily obtained by solving $\tilde{\mathbf{H}}_{0} \underline{u}(i)=y(i)_{P}$, through back substitution. Since $\tilde{\mathbf{H}}_{0}^{-1}$ is also LTT [9], $\tilde{\mathbf{H}}_{0}^{-1} y(i)_{P}$ is equivalent to filtering unblocked $y(i)_{P}$ by the entries in the first column of $\tilde{\mathbf{H}}_{0}^{-1}$ (say $\left\{a_{n}\right\}_{n=0}^{P-1}$ ) and retaining the first $P$ outputs. $\left\{a_{n}\right\}_{n=0}^{P-1}$ can be shown to be the first $P$ impulse response co-efficients of $(1) /(H(z))$. In the presence of noise, due to the implicit filtering involved, such an equalization is viable only for minimum phase $H(z)$ (i.e., stable $(1) /(H(z))$ ). If $H(z)$ were to be maximum phase, the above approach results in undue noise amplification. Use of time reversed filtering instead of the normal filtering, effectively converts $H(z)$ to a minimum phase function, thereby preventing excess noise amplification. The details of time reversed filtering and the approach for a mixed phase system are mentioned below.

Consider a channel with no roots on the unit circle in a noiseless setting. The case of zeros on the unit circle is dealt later. In the presence of noise, Min-max equalization scheme does not implement the SZFE. The performance of this scheme vis-a-vis that of SZFE is discussed in Section V. Let $\left\{f_{n}\right\}_{n=0}^{L_{\max }}\left(H_{\max }(z)\right)$ and $\left\{g_{n}\right\}_{n=0}^{L_{\min }}\left(H_{\min }(z)\right)$ denote the co-efficients (transfer functions) of maximum and minimum phase parts of the channel respectively. Then $H(z)=H_{\min }(z) H_{\max }(z), L=L_{\min }+L_{\max }$. Let $\underline{x}(i)$ be the $N$-fold blocked input with a zero pad of length $L(\underline{u}(i)+L$ zeros) for block transmissions over $H_{\max }(z)$ and let $y_{\max }(i)$ be the corresponding $N$-fold blocked output. Then $\underline{y}_{\max }(i)$ is the input for block transmission over $H_{\min }(z)$ and let $y_{\text {out }}(i)$ be the corresponding $N$-fold blocked output. Since $\underline{x}(n)$ and $\underline{y}_{\max }(n)$ have $L$ and $L_{\min }$ trailing zeros respectively, output at each stage is free from IBI. Let $\mathbf{H}_{\max }$ be the $N \times N$ LTT matrix with $\left[f_{0}, f_{1}, \ldots, f_{L_{\max }}, \ldots, 0\right]_{N \times 1}^{T}$ being its first column. $\mathbf{H}_{\min }$ is the $N \times N$ LTT matrix with $\left[g_{0}, g_{1}, \ldots, g_{L_{\min }}, \ldots, 0\right]_{N \times 1}^{T}$ being its first column. Then,

$$
\underline{y}_{\max }(i)=\mathbf{H}_{\max } \underline{x}(i) ; \quad \underline{y}_{\text {out }}(i)=\mathbf{H}_{\min } \mathbf{H}_{\max } \underline{x}(i) .
$$

It can be shown that $\underline{y}_{\text {out }}(i)$ equals $\underline{y}(i)$ of (1) (noiseless case). Hence we will use $\underline{y}(i)$ instead of $\underline{y}_{\text {out }}(i)$.

\section{A. Equalizer for $\tilde{\mathbf{H}}_{\min }$}

Let $\mathbf{I}_{K}$ and $\mathbf{0}_{N \times K}$ denote the $K \times K$ identity matrix and the $N \times K$ zero matrix respectively. 
Let $\underline{\tilde{y}}_{\max }(i)=\left[\mathbf{I}_{P+L_{\max }} \mid \mathbf{0}_{\left(P+L_{\max }\right) \times L_{\min }}\right] \underline{y}_{\max }(i)$, $\underline{\tilde{y}}(i)=\left[\mathbf{I}_{P+L_{\max }} \mid \mathbf{0}_{\left(P+L_{\max }\right) \times L_{\min }}\right] \underline{y}(i)$ and $\tilde{\mathbf{H}}_{\min }$ be the $\left(P+L_{\max }\right) \times\left(P+L_{\max }\right)$ leading LTT sub-matrix of $\mathbf{H}_{\min }$. Then,

$$
\underline{\tilde{y}}(i)=\tilde{\mathbf{H}}_{\min } \underline{\tilde{y}}_{\max }(i)
$$

Since $\left|g_{0}\right|>0$ (minimum phase property), $\tilde{\mathbf{H}}_{\min }$ is invertible. Hence, (4) can be easily solved for $\tilde{\tilde{y}}_{\max }(i)$ by back substitution. As $\underline{y}_{\max }(i)$ has $L_{\min }$ trailing zeros, recovering $\underline{\tilde{y}}_{\max }(i)$ performs equalization for the minimum phase part.

\section{B. Equalizer for $\tilde{\mathbf{H}}_{\max }$}

Let $\underline{\hat{y}}_{\max }(i)=\left[\mathbf{0}_{P \times L_{\max }} \mid \mathbf{I}_{P}\right] \underline{\tilde{y}}_{\max }(i)$ and $\tilde{\mathbf{H}}_{\max }$ be the $P \times P$ UTT (Upper Triangular Toeplitz) matrix with $\left[f_{L_{\max }}, f_{L_{\max }-1}, \ldots, f_{1}, f_{0}, \ldots, 0\right]_{1 \times P}$ being its first row. Then,

$$
\underline{\hat{y}}_{\max }(i)=\tilde{\mathbf{H}}_{\max } \underline{u}(i)
$$

Since $\left|f_{L_{\max }}\right|>0$ (order: $L_{\max }$ ), $\tilde{\mathbf{H}}_{\max }$ is invertible. Hence (5) can be easily solved for $\underline{u}(i)$ by back substitution, thereby recovering the transmitted symbols. Writing the entries of $\underline{\hat{y}}_{\max }(i)$ in (5) in reverse order, we see that the time reversed input is pumped on the time reversed channel $\left(\left\{f_{L_{\max }-n}\right\}_{n=0}^{L_{\max }}\right)$ to obtain time reversed output (time reversed filtering). Note that the filter $\left\{f_{L_{\max }-n}\right\}_{n=0}^{L_{\max }}$ is minimum phase. As a result, in the noisy case, the otherwise excess noise amplification due to truncated causal filtering by $(1) /\left(H_{\max }(z)\right)$ is tackled. A similar idea is used in [9] for direct blind equalization. The order of equalization can be interchanged and equalizers for the new order can be similarly obtained. Unless specified otherwise, the order is as detailed above.

\section{Zeros on Unit Circle}

Adding zeros on the unit circle (say $L_{z}$ of them) to $H_{\min }(z)$ or/and $H_{\max }(z)$ will not change the nonzero property of the resulting $g_{0}$ and $f_{L_{\max }}$. Hence the earlier process can be used with modified $L_{\min }$ and/or $L_{\max }$. Thus Min-max equalization possesses the ZFEQN property for all channels. However, in the noisy case, it is seen that as $L_{z}$ increases, performance of this scheme departs from that of SZFE. When zeros are present on the unit circle, SZFE can be used instead. However, for low $L_{z}$, (say $L_{z}<P / 20$ ), gains obtained by using SZFE are small. We club the $L_{z}$ zeros on unit circle to $H_{\max }(z)$ in all further discussions.

\section{Computational Complexity of Min-Max Equalizer}

1) Finding the channel zeros: The roots of $H(z)$ are computed as the eigen-values of the companion matrix formed by $\left\{h_{n}\right\}_{n=0}^{L}$ [3] (roots command of Matlab). We approximate the complexity as $O\left(L^{3}\right) \mathrm{CA}$ and $O\left(L^{3}\right) \mathrm{CM}^{2}$. LAPACK manual notes the complexity as $10 L^{3}$. Better root-

\footnotetext{
${ }^{2}$ This involves Balancing, Hessenberg reduction followed by QR iterations to obtain the Schur form. Each iteration results in $O\left(L^{2}\right)$ operations and the maximum number of iterations is a function of $L$
}

finding algorithms and their computations are presented in the survey paper [8].

2) Obtaining $\left\{g_{n}\right\}$ and $\left\{f_{n}\right\}$ ( $H_{\max }(z)$ includes zeros on unit circle): Requires $O\left(L^{2}\right) \mathrm{CA}$ and $O\left(L^{2}\right) \mathrm{CM}$.

3) Back substitutions (1) For $\tilde{\mathbf{H}}_{\min }:\left(P+L_{\max }-\right.$ $\left.L_{\min }\right) L_{\min }+\left(L_{\min }\left(L_{\min }-1\right)\right) /(2) \mathrm{CM},\left(P+L_{\max }-\right.$ $\left.L_{\min }\right) L_{\min }+\left(L_{\min }\left(L_{\min }-1\right)\right) /(2) \mathrm{CA}$ and $P+L_{\max } \mathrm{CD}$. (2) For $\tilde{\mathbf{H}}_{\max }:\left(P-L_{\max }\right) L_{\max }+\left(L_{\max }\left(L_{\max }-1\right)\right) /(2)$ $\mathrm{CM},\left(P-L_{\max }\right) L_{\max }+\left(L_{\max }\left(L_{\max }-1\right)\right) /(2) \mathrm{CA}$ and $P$ CD. The low bandwidths of $\tilde{\mathbf{H}}_{\max }$ and $\tilde{\mathbf{H}}_{\text {min }}$ are used here. Hence the approximate complexity is $O\left(L^{3}\right)+O(P L)$ flops. Depending on the computations for back-substitutions, order of equalization can be interchanged to get further savings.

\section{ChOICE OF THE Algorithm}

\section{A. Metric 1: Computational Complexity}

Table I lists the approximate complexities of the three schemes. We now try to find values of $L$ (given a $P$ ) for which a given algorithm is cheaper. Finding such $L$ by comparing actual complexities listed in Sections III and IV is cumbersome. Instead, an analysis based on Table I is used to find the approximate values of $L$ for which an algorithm is cheaper. Simulations are carried out to obtain numerical values of the flops required by each of the three schemes. These simulations aid the analysis in refining the earlier obtained values of $L$. We use "flops" command of Matlab Version 5.3 for counting operations in the simulations. Adjustments are made to the flop counts to account for real-complex operations. Since computations for min-max scheme depend on the actual channel (via $L_{\max } \& L_{\min }$ ), average flop count obtained over 1000 realizations of a random channel is presented. We present the flop count ratios (for clarity) in Table II for a few $(P, L)$.

\section{B. Metric 2: BER Performance}

Since BBH and Householder's methods are equivalent implementations of the SZFE, their BER curves coincide (the numerical properties of the two algorithms are assumed to be similar). Hence, we present the BER curve of BBH method and refer it to as the BER curve of SZFE. BER performance of Min-max equalization is different from that of SZFE and needs to be presented. The BER curves are plotted in Figs. 1(a) and (b) for the HiperLAN/2 channel models B and E respectively. QPSK modulation and $P=64$ are used. BER curves are based on Monte Carlo simulations with each trial corresponding to a different realization of the channel used. The channel is fixed for a frame (training symbols $+\underline{u}(i)+L$ zeros) and varies across frames. The channel is assumed to be estimated perfectly by the receiver in every frame. At low values of $E_{b} / N_{0}$ (till 5-8 dB), the performance of Min-max equalizer is similar to that of SZFE. For $E_{b} / N_{0}$ in the range 8-15 dB, Min-max equalizer requires about $1-1.5 \mathrm{~dB}$ of extra signal energy to have a similar BER as SZFE. This offset rises to about $2-3 \mathrm{~dB}$ for $E_{b} / N_{0} \approx 25 \mathrm{~dB}$. We now evaluate the "attractiveness" of the schemes described.

1) BBH Algorithm: For a given $P$, Table I shows that the approximate complexity of BBH increases linearly (nearly) with $L(L<P)$, while the increase is quadratic or cubic for the 
TABLE I

SUMMARY OF APPROXIMATE FLOPS (A.F) OF VARIOUS SCHEMES

\begin{tabular}{c|c|c|c|c|c}
\hline Scheme & A.F & Scheme & A. F & Scheme & A. F \\
\hline BBH & $O\left(P^{2}\right)+O(P L)$ & Householder's & $O\left(P L^{2}\right)+O(P L)$ & Min-Max & $O\left(L^{3}\right)+O(P L)$ \\
\hline
\end{tabular}

TABLE II

Flop Count Ratios of VARious Schemes For DifFerent $(P, L) \cdot R_{1}=$ Flop Count of Householder's Method/Average Flip Count of Min-Max Equalization. $R_{2}=$ Flop Count of BBH Algorithm/Average Flop Count of Min-Max Equalization

\begin{tabular}{c|c|c|c|c|c|c|c|c}
\hline$(P, L)$ & $R_{1}$ & $R_{2}$ & $(P, L)$ & $R_{1}$ & $R_{2}$ & $(P, L)$ & $R_{1}$ & $R_{2}$ \\
\hline$(64,1)$ & 5.7477 & 86.7485 & $(128,1)$ & 5.8419 & 168.9554 & $(512,4)$ & 9.1523 & 251.3249 \\
$(64,2)$ & 6.9665 & 61.9713 & $(128,2)$ & 7.1438 & 120.7644 & $(512,6)$ & 9.8382 & 148.5423 \\
$(64,6)$ & 2.6364 & 6.5196 & $(128,8)$ & 3.7997 & 10.1474 & $(512,12)$ & 8.2971 & 37.5450 \\
$(64,8)$ & 2.0519 & 3.2227 & $(128,11)$ & 2.6093 & 3.6803 & $(512,22)$ & 5.0082 & 6.8584 \\
$(64,12)$ & 1.2231 & 1.1133 & $(128,19)$ & 1.5769 & 1.0531 & $(512,49)$ & 2.6440 & 1.0230 \\
$(64,13)$ & 1.1526 & 0.9059 & $(128,20)$ & 1.5314 & 0.9948 & $(512,50)$ & 2.6122 & 0.9763 \\
$(64,20)$ & 0.6783 & 0.3026 & $(128,24)$ & 1.2912 & 0.6190 & $(512,51)$ & 2.5489 & 0.9208 \\
\hline
\end{tabular}

other schemes. Hence BBH algorithm is suited for large $L$ (i.e., $L$ such that $L^{3}>\alpha P^{2}$, for some $\alpha>0$ ). Low value of $R_{2}$ in Table II for $(64,13),(128,20)$, and $(512,50)$ shows that $\alpha$ can be chosen as $0.5{ }^{3}$ Since OFDM systems prefer a low $(L / P)$, scope of this algorithm being attractive is limited.

2) Householder's Method: From Table I we infer that Householder's method would be cheaper than BBH method for $L^{2}<\beta P$ for some $\beta>0$. Comparing the values of $R_{1}$ and $R_{2}$ in Table II and other simulations, we choose $\beta=1$. For small values of $L, R_{1}$ increases with $L$ as rooting is cheap in the min-max scheme. For larger $L$, computations for rooting becomes dominant and $R_{1}$ decreases with increase in $L$. Further, Table II and other simulations show that Householder's method hardly beats Min-max scheme in computations for $L<\sqrt{P}$. However, the BER advantage [Figs. 1(a) and (b)] of this method, especially at high $E_{b} / N_{0}$, should be weighed against computational simplicity of Min-max equalization. This results in a BER-Computation tradeoff, wherein the algorithm needs to chosen based on system considerations.

3) Min-Max Equalization: For very small $L$, rooting is accurate and cheap [8]. For such $L$, this scheme requires about $O(P L)$ flops, which is less than the flops required by other algorithms $\left(O\left(P L^{2}\right), O\left(P^{2}\right)\right)$. Further Tables I, II, Section V.C.1 and simulations show that min-max method is cheaper for $L$ slightly less than $\left(0.5 P^{2}\right)^{1 / 3}$. However, BER performance should also be evaluated to favor this algorithm. Figs. 1(a) and (b) show that for low $E_{b} / N_{o}$, the BER performance of this scheme is similar to that of SZFE. Thus for low $L$ and low $E_{b} / N_{o}$, Min-max equalization is attractive.

\section{CONCLUSION}

In this work, we have used QR Decomposition to obtain low complexity implementations of SZFE for the ZP scheme. In particular, BBH and Householder's algorithms are used. Motivated by equalization using FIR filters, we have developed the Min-Max equalization scheme which yields a channel independent ZFE. The computational complexity and BER performance of each of these schemes are evaluated. While BBH is the best for large $L$, Min-max equalization is best for low

${ }^{3}$ While the Nagy's algorithm [4], [5] is cheaper (by about $\left.\left(P^{2} / 2\right) \mathrm{CA} / \mathrm{CM}\right)$ in computing of $\mathbf{Q}^{*} y(i)$ than BBH, its flop count can be shown to be $O\left(P^{2}\right)+O(P L)$. Using Nagy's algorithm instead of $\mathrm{BBH}$, will only cause a small change in $\alpha$ without altering the spirit of the discussions.

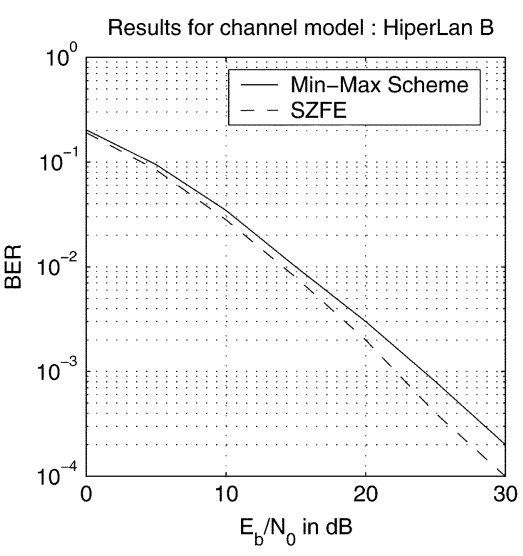

(a)

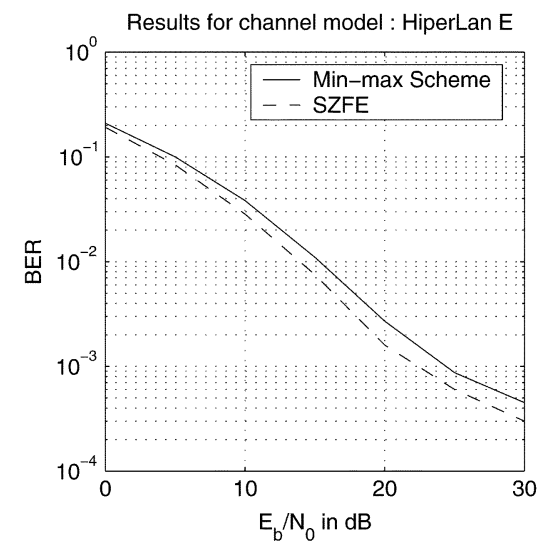

(b)

Fig. 1. BER Curves for HiperLAN/2 Channel models (a) B. (b) E.

$L$ and low $E_{b} / N_{o}$. For large $E_{b} / N_{o}$ and small $L$, we have a BER-complexity trade-off between Householder's method and the Min-max scheme. The performance of these methods for imperfect channel estimates using their numerical properties [3], [7], [8] needs a detailed study.

\section{REFERENCES}

[1] G. B. Giannakis and Z. Wang, "Wireless multicarrier communications-Where fourier meets shannon," IEEE Signal Processing Mag., pp. 29-48, May 2000.

[2] B. Muquet, M. de Courville, G. B. Giannakis, Z. Wang, and P. Duhamel, "Reduced complexity equalizers for zero-padded OFDM transmissions," in Proc. ICASSP, vol. 5, Istanbul, Turkey, 2000, pp. 2973-2976.

[3] G. H. Golub and C. F. Van Loan, Matrix Computations. London, U.K.: The Johns Hopkins University Press, 1996.

[4] J. G. Nagy, "Fast inverse QR factorization for Toeplitz matrices," in SIAM J. Sci. Comput., vol. 14, Sept. 1993, pp. 1174-1193.

[5] C. V. Sinn, F. Hanschmann, and J. Gotze, "Efficient algorithms relating OFDM channel parameters to filter weights in single carrier systems with frequency domain equalization," in Proc. 7th Int. OFDM Workshop, Hamburg, Germany, Sept. 2002.

[6] A. W. Bojanczyk, R. P. Brent, and F. R. de Hoog, "Linearly connected arrays for Toeplitz least squares problems," J. Parallel Distrib. Comput., vol. 9, pp. 261-270, 1990.

[7] R. P. Brent, "Stability of fast algorithms for structured linear systems," Fast Reliable Algorithms for Matrices with Structure, pp. 103-116, 1999.

[8] V. Y. Pan, "Solving a polynomial equation: Some history and recent progress," in SIAM Rev., vol. 39, June 1997, pp. 187-220.

[9] A. Scaglione, G. B. Giannakis, and S. Barbarossa, "Redundant filterbank precoders and equalizers part II: Blind channel estimation, synchronization and direct equalization," IEEE Trans. Signal Processing, vol. 47, pp. 2007-2022, July 1997. 